\title{
Classification of Retinal Pathologies using Convolutional Neural Network
}

\author{
S. Amaladevi ${ }^{1}$, Dr. Grasha Jacob ${ }^{2}$ \\ ${ }^{1}$ Research Scholar, Manonmaniam Sundaranar University, Abishekapatti, \\ Rani Anna Government College for Women, Tirunelveli 627 012, TamilNadu, India, rshaamlu77@gmail.com \\ ${ }^{2}$ Associate Professor, Research Department of Computer Science,Rani Anna Government College for Women, \\ Tirunelveli 627 008, Tamil Nadu, India, grasha.amala@gmail.com
}

\begin{abstract}
Deep Learning technique has emerged as a successful approach in applications with complex functions. It is used in the areas of computer vision, speech recognition and image processing applications. The most popular deep learning architecture is Convolution Neural Network (CNN). The proposed method a pre-trained CNN based on GoogLeNet and VGGNet is used to classify the retinal diseases such as Age-Related Macular Degeneration (AMD) and Diabetic Macular Edema (DME). Spectral Domain Optical Coherence Tomography (SD-OCT) images are used for identifying the retinal pathologies. Pre-trained GoogLeNet and VGGNet predict the nature of all the subjects of the test images. 94\% of accuracy, 95\% specificity and 96\% sensitivity for the three classes are achieved using GoogLeNet and $96 \%$ of accuracy, $99 \%$ specificity and $96 \%$ of sensitivity for the three classes are achieved using VGGNet.
\end{abstract}

Key words : Age-related Macular Degeneration, Convolutional Neural Network, Diabetic Macular Edema, GoogLeNet, Spectral Domain Optical Coherence Tomography, VGGNet.

\section{INTRODUCTION}

Spectral Domain Optical Coherence Tomography (SD-OCT) is a non-invasive, imaging modality for ophthalmology to detect the earlier stage of the retinal pathologies through the variations in texture and morphology [4]. This can be used to acquire high resolution cross-sectional visualization of the morphology of retina as well as the retinal layers[6]. The World Health Organization (WHO) said 30 million of people were affected by diabetes in 1985, and in 2000 the number

Retinal detachment can occur when too much of fluid is accumulated behind the retina causing division [15]. The result of the deformation and detachment of the subsequent layers is vision loss. Early detection and diagnosis of the deformation, fluid fill, drusen and geographic atrophy prevent vision loss[5]. The diagnosis of these pathology is based on the visual clinical examination of the SD-OCT images by the well-trained ophthalmologists. To increase the speed of the visual investigation process, the system is divided into solvable small units. This process will reduce had increased to 177 million worldwide. The WHO announced that this would increase to 370 million people in 2030 [16].

Human retina is most important part and affected by diabetics [12]. The retinal tissue consists of layers in the back of the eye that senses light and sends signals to our brain. Retinal disorders affect this vital tissueto cause blindness. Eye diseases such as Diabetic Retinopathy (DR)[7], its complications, Diabetic Macular Edema (DME) [14], and Age-related Macular Degeneration (AMD) are the most common causes of irreversible vision loss in individuals with diabetes [11]. The ophthalmology diagnosis system is based on the patterns of AMD, DME image slices and clinical guidelines. Figure 1(a). shows the normal macula with clear boundaries between layers. Figure 1(b). shows AMD retina, drusen is accumulated between chorid and retinal pigment epithelium (RPE) layer.Geographic atrophy (GA)is the advanced form of AMD, which is frequently associated with loss of central vision [3]. Figure 1(c), shows the DME retina, micro vascular is changed in the retina that develops the thickness in the basement membrane. The liquid is accumulated in the vascular walls to form the black blobs in the images.

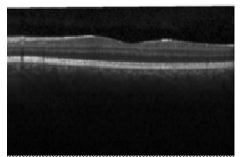

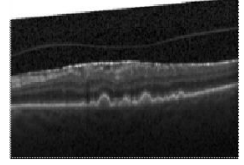

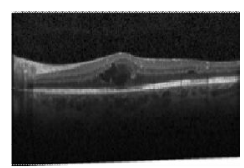

Figure 1: Retinal Layers (a)Normal, (b)AMD and (c)DME

the burden of ophthalmologist. The solvable small units are preprocessing of the image, feature extraction, and classification. Convolutional Neural Network (CNN) is the most important technique of artificial neural network for image classification system [17] which is composed of multiple layers to learn features in multiple levels [9] and mostly used in image analysis applications [13]. CNN consists of different types of layers formed in a hierarchal manner. These layers are convolutional layer with learnable filters (conv), pooling layer (pool), fully connected (FC) 
layer with dropout and softmax layer [9]. Convolutional layer is used to generate a feature map. Pooling layer is used to reduce the spatial size of the activation map. The fully connected layer computes class scores. The dropout is used to reduce the overfitting in fully connected layer [8]. The softmax layer converts the output of the final layer into class probabilities. In each layer a learnable feature is transformed into next layer. Finally, the results of the classified images are obtained.

In the classification system, Convolutional Neural Network based pre-trained GoogLeNet and VGGNet architectures are used to classify the images and analyse the result.Figure 2 shows the convolutional neural network architecture.

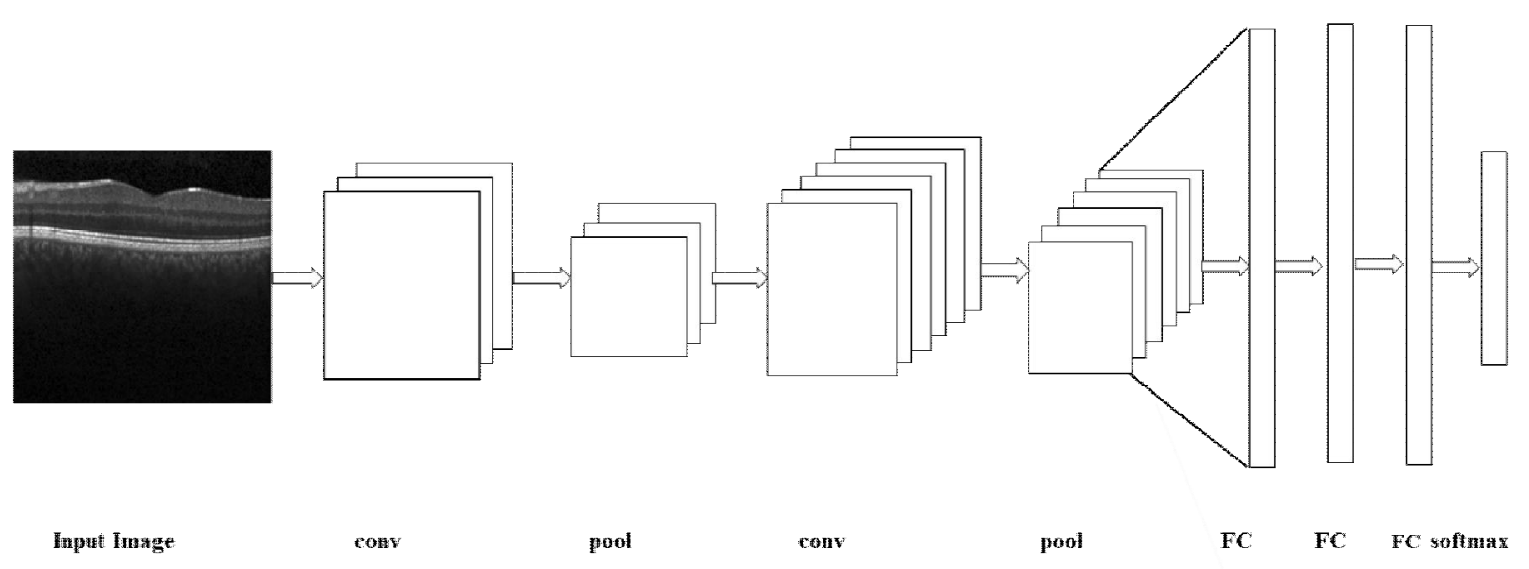

Figure 2: Convolutional neural network architecture

\section{LITERATURE REVIEW}

A number of algorithms were proposed to simplify the diagnosis process of retinal diseases. Lemaitre et al.[10] have discussed a methodology for automatic identification of normal and DME patients. In this method non-local mean (NLM), flattening, and slice alignment techniques were used for enhancing the OCT volumes. Local and Global mapping for different sampling points were used to extract the Local Binary Patterns (LBP) features and five different classifiers (K-Nearest Neighbor (KNN)), Logistic Regression (LR), Random Forest (RF) and Gradient Boosting (GB) and Support Vector Machine (SVM) were used to classify DME patients. Wang et al. [21] have identified a machine learning basedcomputer-aided diagnosis (CAD) model that used a Correlation-based Feature Subset (CFS) algorithm to extract the Linear Configuration Pattern (LCP) features. The quadratic programming-based algorithm Sequential Minimal Optimization (SMO) was used to classify the AMD and DME retinal diseases.Srinivasan et al.[19] have proposed a novel algorithm for detection of retinal diseases. In this method, sparsity- based block matching and 3D-filtering (BM3D) method was used to remove the speckle noise and flattening the natural retinal curvature to reduce the variations in SD-OCT image slices. The multiscale histogram of oriented gradient (HOG) feature was extracted and linear support vector machine was used to classify the normal, age-related macular degeneration (AMD), and diabetic macular edema (DME) retinal diseases.

Zhang et al. [22] have implemented an ensemble of one-class kernel for classification of medical images. Features were extracted from the training images. One type of feature was trained in each one-class classifier. The kernel principal component analysis (KPCA) was used for base classifier. Albarrak et al. [1] have proposed to build an automated diagnostic system of AMD for 3D OCT retinal volume. In this system every preprocessed volume of images were decomposed into a set of sub volumes. Oriented gradient Local Binary Pattern histogram feature was generated in each sub volume. The combination of decomposition and LBP histogram were used to generate an accurate feature. Bayesian network classifier was used to classify the feature vectors. Anantrasirichai et al. [2] have discussed a Support Vector Machine classifier to detect glaucoma. In this system, the inner retinal layer thickness (ganglion cell complex) and texture features (RLM, GLCM, CWT, LBP, GRA) were extracted and Principal component analysis method was used to improve the accuracy.

Machine learning models based computational algorithms learn the features directly from data and require large amounts of data during the experiment. The performance of a machine learning method was mostly based on the choice of the extracted data feature. During pre-processing, an effective method was required to achieve the best result. Depth plays an important role during the learning process. Deep Learning (DL) method is similar to the behaviour of humans- to learn the features from experience. Therefore, a proper understanding of the model is essential. The best performance can be achieved by the repeated experimentation of the trained model.

It is evident that deep learning based pretrained GoogLeNet and VGGNet can be used to classify the SD-OCT images of age-related macular degeneration (AMD), diabetic macular edema (DME). 


\section{METHODOLOGY}

The proposed system consists of several stages of the process to detect the retinal diseases of SD-OCT images. The proposed architecture of the system is shown in Figure 3.

The first step is pre-processing. During pre-processing, sparsity-based block matching and 3D filtering (BM3D) denoising method are applied to remove the speckle noise.
The natural curvature of the retinal image slice is varying from one person to another. Every image slice is flattened to remove the invariance to clearly identify Retinal Pigment Epithelium (RPE) layer. The flattened images are then resized to $224 \times 224$. The pre-processed images are transformed into the GoogLeNet and VGGNet to classify the image into normal, AMD and DME retina.

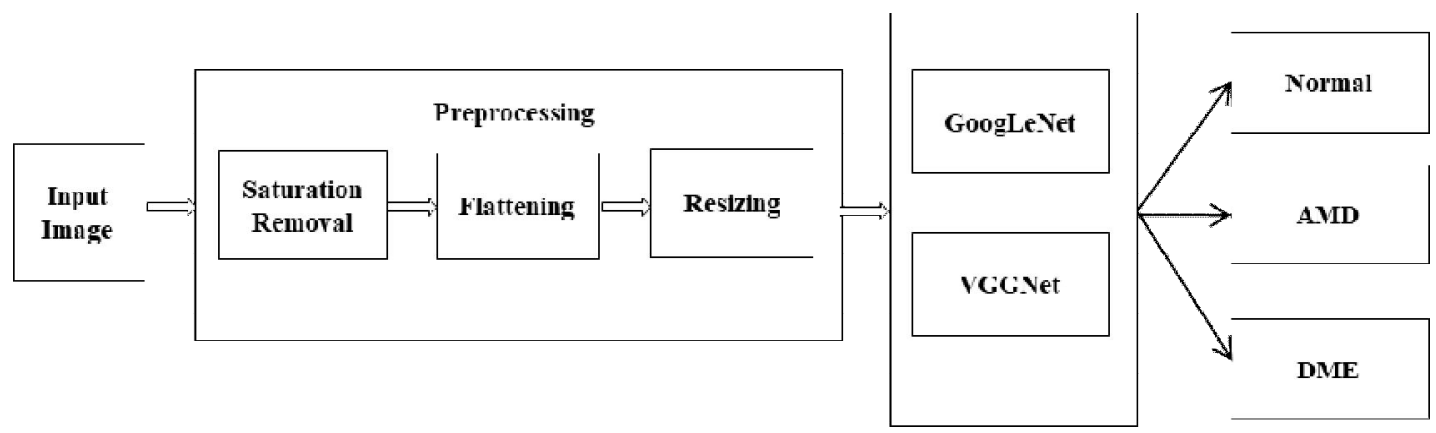

Figure 3: Architecture of the system

\subsection{GoogLeNet}

A pre-trained GoogLeNet model is 22 layers deep [20]. This CNN based architecture consists of repeated number of the convolution block, maxpooling block, activation block, and inception layer. The inception layer is a small network. It has a more complex structure. Inside the structure, the Rectified Linear Unit (ReLU) is appended to convolution blocks. During the processing time, a potential response will be identified at every layer. The pre-trained GoogLeNet predict the test images as normal, AMD and DME.

\subsection{VGGNet 16}

VGGNet-16 is more suitable for classification and feature extraction. VGGNet-16 has 16 layers [18]. There are 13 convolutional layers, 3 fully connected layers and one softmax layer. In the network system, all convolutional layers have filters of size 3-by-3. The most important feature of the design is depth. Increase in the depth of the network is achieved by adding more convolution layers. The input size of an image for this model is $224 \times 224 \times 3$. The image is passed through a stack of convolution layers. It uses $3 \times 3$ convolution filters throughout the network. Max pooling is 2 by 2 pixel window with a stride of 2 , then another stack of convolutional layers followed by three fully connected layers. The VGGNet predicts the test images as normal, AMD and DME.

\section{EXPERIMENTAL RESULT}

The Duke University SD-OCT dataset[19] was used for classification of retinal images in the proposed system. This dataset consists of data from 45 participants, 15 participants with normal, 15 participants with AMD, and 15 participants with DME retina. Multiple images exist for each participant.The CNN architecture consists of training and testing phase. The set of SD-OCT images and their labels are defined by $\left(\mathrm{I}_{\mathrm{m}} \subset\right.$ \{normal, AMD, DME $\left.\}\right)$. These images are used as training and testing data. The pre-trained GoogLeNet and VGGNet are designed for $224 \times 224$ images, with three channels. The SD-OCT images are pre-processed, selfreplicated three times and concatenated to be $3 \times 224 \times 224$. Figure 4 shows the 2D - horizontal cross-sectional retinal input images of normal, AMD, and DME.

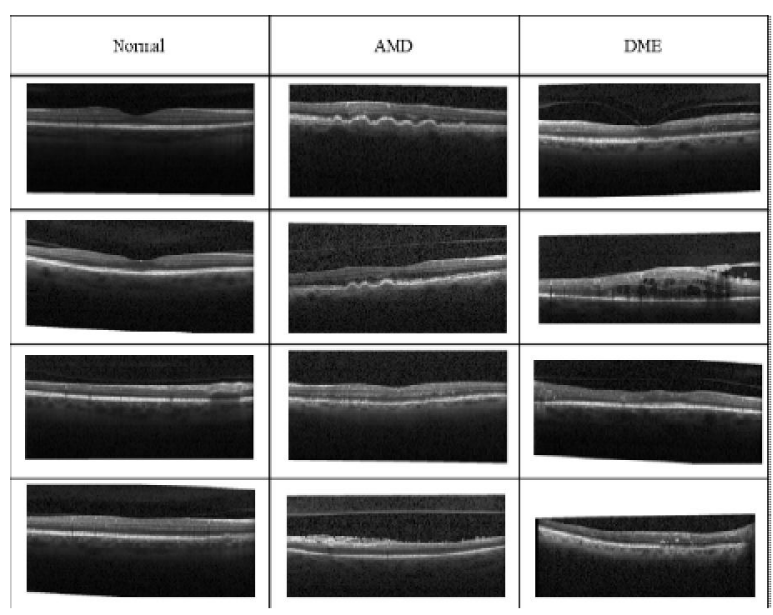

Figure 4: Retinal images of normal, AMD and DME

During the training phase 24 images are selected (8 each from normal, AMD and DME class). Mean image of the training set is calculated and it is subtracted from each image of the training set. The training data is divided into batches and every batch has 10 images. Each epoch is treated as an experiment. An experiment is defined as 50 epoch and is used to compute the prediction. 
During the testing phase, the remaining 21 images are selected (7 each from normal, AMD and DME). Training set mean image is subtracted from each test image. The Pretrained GoogLeNet and VGGNet are used to predict the feature of all the subjects of the test image. During processing, different size kernels are applied to the convolution layer to produce the image feature map. This map is used to easily identify the deformations in the RPE layer. Rectified Linear Unit (ReLU) removes negative responses from the feature map. Max pooling layer is used to identify the spatial invariance of feature map by assigning the maximum response to all neighboring pixels and applying the stride to reduce the image size. The LRN1 is used to renormalize the image and to boost the responses. The fully connected layer is used to extract the abstractions of feature map. The softmax layer is used to classify the retinal pathology.

The performance measurement of the system is estimated by the values of accuracy, sensitivity and specificity. Proposed system is three-class classification system. In the three-class classification system sensitivity and specificity are measured by average of the three classes. Accuracy is defined as the ratio of the number of correctly predicted labels divided by the total number of labels. Sensitivity is defined as the number of true positive values divided by the sum of the number of true positives and the number of false negatives. Specificity is defined as the number of true negatives divided by the number of negatives. The overall performance of the classification system is measured based on the indicators of sensitivity, specificity and accuracy and these results are clearly presented in the Table 1.

Table 1: Performance measure of GoogLeNet and VGGNet

\begin{tabular}{|c|c|c|}
\hline & G00gLcNct & VGGNct \\
\hline Sensittvity & 0.9651 & 0.9620 \\
\hline Specificity & 0.9503 & 0.9908 \\
\hline Accuracy & 0.9427 & 0.9620 \\
\hline
\end{tabular}

With GoogLeNet 94\% of accuracy, 95\% specificity, and $96 \%$ of sensitivity of the three classes is achieved. Using VGGNet $96 \%$ of accuracy, 99\% specificity and 96\% sensitivity for the three classes is achieved.It is clear that VGGNet has achieved significant result than the GoogLeNet. Figure 5 shows the training accuracy GoogLeNet and VGGNet.

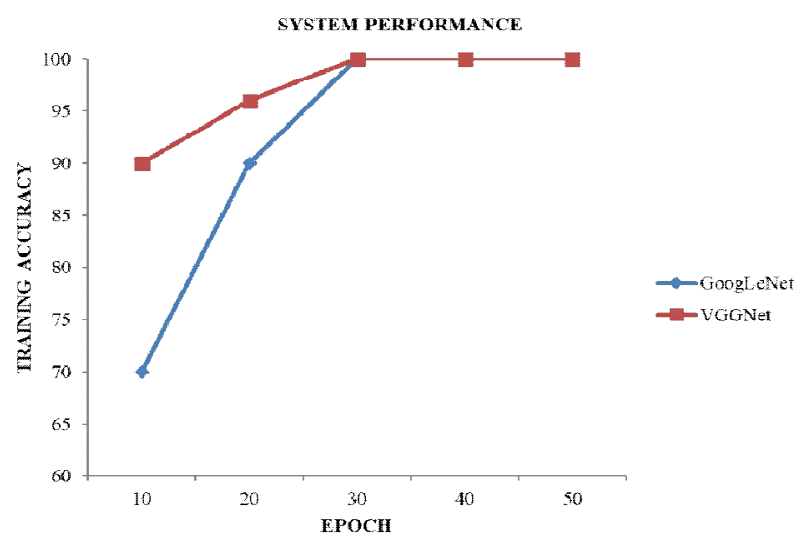

Figure 5: Training Accuracy of GoogLeNet and VGGNet

This line graph clearly shows that the VGGNet has achieved better performance compared to GoogLeNet.

\section{CONCLUSION}

In this work, two different types of Convolutional Neural Network (CNN) architectures of pre-trained GoogLeNet and VGGNet are used for retinal image classification. These systems used the Spectral domain optical coherence tomography (SD-OCT) images to classify the retinal diseases, such as diabetic macular edema and age-related macular degeneration. $94 \%$ of accuracy, $95 \%$ specificity and 96\% sensitivity for the three classes are achieved using GoogLeNet and $96 \%$ of accuracy, 99\% specificity and $96 \%$ of sensitivity for the three classes are achieved using VGGNet. VGGNet has achieved betterperformance compared to GoogLeNet.

\section{REFERENCES}

1. A. Albarrak, F. Coenen, and Y. Zheng, Age-related Macular Degeneration Identification in Volumetric Optical Coherence Tomography Using Decomposition and Local Feature Extraction, proceedings of the $17^{\text {th }}$ Annual Conference in Medical Image Understanding and Analysis (MIUA),pp. 5964, 2013.

2. N. Anantrasirichai, A. Achim, J.E. Morgan, I. Erchova and L. Nicholson, SVM-based texture classification in Optical Coherence Tomography, proceedings of the IEEE 10th International Symposium on Biomedical Imaging (ISBI), pp. 13321335, 2013. https://doi.org/10.1109/ISBI.2013.6556778

3. C. Bowes Rickman, S. Farsiu, C. A. Toth, and M. Klingeborn, Dry Age-Related Macular Degeneration: Mechanisms, Therapeutic Targets, and Imaging, Invest. Ophthalmol. Vis. Sci.Vol. 54, No.14, pp. 68-80,2013. https://doi.org/10.1167/iovs.13-12757

4. B. Cense, N. Nassif, T. Chen, M. Pierce, S.H. Yun, B. Park, B. Bouma, G. Tearney, and J. De Boer, Ultra high resolution high-speed retinal imaging using 
spectral-domain optical coherence tomography, Opt. Express, Vol. 12, No. 11, pp. 2435-2447, 2004.

5. S.J. Chiu, J.A. Izatt, R. V. O 'Connell, K. P. Winter, C. A. Toth, and S. Farsiu, Validated Automatic Segmentation of AMD Pathology Including Drusen and Geographic Atrophy in SD-OCT Images, Invest. Ophthalmol. Vis. Sci.Vol. 53, pp. 5361, 2012.

https://doi.org/10.1167/iovs.11-7640

6. W. Drexler and J.G. Fujimoto, state-of-the-art retinal optical coherence tomography, Prog. Retin. Eye Res. Vol. 27, No. 1, pp. 45-88, 2008.

7. D.K. Kirange, J.P. Chaudhari, K.P. Rane, K.S. Bhagat, Nandinichaudhri, Diabetic Retinopathy Detection and Grading Using Machine Learning, International Journal of Advanced Trends in Computer Science and Engineering, Vol 8, No.6, pp. 3570-3576, 2019. https://doi.org/10.30534/ijatcse/2019/139862019

8. A. Krizhevsky, I. Sutskever and G.E. Hinton, ImageNet Classification with Deep Convolutional Neural Networks, Adv. Neural Inf. Process. Syst. pp. 1097-1105, 2012.

9. Y. LeCun, Y. Bengio and G. Hinton, Deep learning, Nature, Vol.521, No.7553, pp.436-444, 2015.

10. G. Lemaitre, M. Rastgoo, J. Massich, C. Y. Cheung, T.Y. Wong, E. Lamoureux, D. Milea, F. Meriaudeau and D. Sidibe, Classification of SD-OCT volumes using local binary patterns: Experimental Validation for DME detection, J. Ophthalmol. 2016. https://doi.org/10.1155/2016/3298606

11. D. Michael Abramoff, Mona k Garvin, Milan Sonka, Retinal Imaging and Image Analysis, IEEE Rev Biomed Eng. Vol 3, pp.169-208, 2010.

12. Navneet Malik, Nilesh N Wani, Jimmy Singla, Complications of Sight Threatening Diabetic Retinopathy: A Review, International Journal of Advanced Trends in Computer Science and Engineering, Vol 8, No.4, pp.1038-1044, 2019. https://doi.org/10.30534/ijatcse/2019/09842019

13. M. Oquab, L. Bottu, I. Laptev, and J. Sivic, Weakly supervised object recognition with convolutional neural networks, Adv. Neural Inf. Process. Syst. pp. 1717-1724, 2014.

14. T. Otani, S. Kishi, and Y. Maruyama, Patterns of diabetic macular edema with optical coherence tomography, Am. J. Ophthalmol. Vol. 127, No. 6, pp. 688-693, 1999.

15.G. Quellec, Kyungmoo Lee, M. Dolejsi, M.K. Garvin, M.D. Abramoff, and M. Sonka,Three-dimensional analysis of retinal layer texture: Identification of fluid-filled regions in SD-OCT of the macula, IEEE Trans onMedical Imaging, Vol. 29, No. 6, pp. 1321 1330, 2010.

16. P. Romero Aroca, Current status in diabetic macular edema treatments, World Journal of Diabetes, Vol. 4, No. 5, pp. 165-169, 2013.

https://doi.org/10.4239/wjd.v4.i5.165
17.J. Schmidhuber Deep learning in neural networks: an overview, Neural Networks, Vol. 61, pp. 85-117, 2015.

18. K. Simonyan and A. Zisserman, Very deep convolutional networks for large-scale image recognition, proceedings of Learning Representation (ICLR), pp. 1409-1556, 2015.

19. P.P. Srinivasan, L. A. Kim, P. S. Mettu, S. W. Cousins, G. M. Comer, J. A. Izatt and S. Farsiu, Fully automated detection of diabetic macular edema and dry age-related macular degeneration from opticalimage classification, coherence tomography images, Biomed.Opt. Express, Vol. 5, No. 10, pp. 3568-3577, 2014. https://doi.org/10.1364/BOE.5.003568

20. C. Szegedy, W. Liu, Y. Jia, P. Sermanet, S. Reed, D. Anguelov, D. Erhan, V. Vanhoucke and A. Rabinvich, Going deeper with convolutions, proceedings of the IEEE conference on Computer Vision and Pattern Recognition, pp. 1-9, 2015.

21. Y. Wang, Y. Zhang, Z. Yao, R. Zhao, and F. Zhou, Machine learning based detection of age-related macular degeneration and diabetic macular edema from optical coherence tomography images, Biomed. Opt. Express, Vol. 7, No. 12, pp. 4928-4940, 2016.

22. Y. Zhang, B. Zhang, F. Coenen, J. Xiao, and W. Lu, One-class kernel subspace ensemble for medical image classification, EURASIP J. Adv. Signal Process. pp. 1-13, 2014. https://doi.org/10.1186/1687-6180-2014-17 